Published in final edited form as:

Psychiatry Res. 2013 January 30; 211(1): 1-10. doi:10.1016/j.pscychresns.2012.07.001.

\title{
Localized differences in caudate and hippocampal shape are associated with schizophrenia but not antipsychotic type
}

\author{
Robert McClure ${ }^{a}$, Martin Stynera,b, ${ }^{\star}$, Eric Maltbie ${ }^{a}$, Jeffrey Lieberman ${ }^{d}$, Sylvain Gouttard ${ }^{\mathrm{e}}$, \\ Guido Gerige, Xiaoyan $\mathrm{Shi}^{\mathrm{c}}$, and Hongtu Zhu ${ }^{\mathrm{C}}$ \\ aDepartments of Psychiatry, University of North Carolina, Chapel Hill, NC, 27599, USA \\ ${ }^{b}$ Computer Science, University of North Carolina, Chapel Hill, NC, 27599, USA \\ 'Biostatistics, University of North Carolina, Chapel Hill, NC, 27599, USA \\ dDepartment of Psychiatry, Columbia University, New York, NY 10032, USA \\ eScientific Computing and Imaging Institute, University of Utah, Salt Lake City, UT 84112, USA
}

\begin{abstract}
Background-Caudate and hippocampal volume differences in patients with schizophrenia are associated with disease and antipsychotic treatment, but local shape alterations have not been thoroughly examined.
\end{abstract}

\begin{abstract}
Methods-Schizophrenia patients randomly assigned to haloperidol and olanzapine treatment underwent MRI scans at 3, 6, and 12 months. The caudate and hippocampus were represented as medial representations (M-reps); mesh structures derived from automatic segmentations of high resolution MRIs. Two quantitative shape measures were examined: local width and local deformation. A novel nonparametric statistical method adjusted exponentially tilted (ET) likelihood, was used to compare the shape measures across the three groups while controlling for covariates.
\end{abstract}

Results-Longitudinal shape change was not observed in the hippocampus or caudate when the treatment groups and controls were examined in a global analysis, nor when the three groups were examined individually. Both baseline and repeated measures analysis showed differences in local caudate and hippocampal size between patients and controls, while no consistent differences were shown between treatment groups.

Conclusions-Regionally specific differences in local hippocampal and caudate shape are present in schizophrenic patients. Treatment related longitudinal shape change was not observed within the studied timeframe. Our results provide additional evidence for disrupted cortico-basal ganglia-thalamo-cortical circuits in schizophrenia.

Clinical trial information-This longitudinal study was conducted from March 1, 1997, to July 31, 2001, at 14 academic medical centers (11 in the United States, 1 in Canada, 1 in the Netherlands, and 1 in England). This study was performed prior to the establishment of centralized registries of federally and privately supported clinical trials.

(c) 2012 Elsevier Ireland Ltd. All rights reserved.

Corresponding Author: Martin Styner, University of North Carolina, CB 7160, Department of Psychiatry, Chapel Hill, NC 27599, Telephone: (919) 843-1092, Fax: (919) 966-7225, martin_styner@ieee.org.

Publisher's Disclaimer: This is a PDF file of an unedited manuscript that has been accepted for publication. As a service to our customers we are providing this early version of the manuscript. The manuscript will undergo copyediting, typesetting, and review of the resulting proof before it is published in its final citable form. Please note that during the production process errors may be discovered which could affect the content, and all legal disclaimers that apply to the journal pertain. 


\section{Keywords}

MRI; schizophrenia; morphometry; caudate; hippocampus; antipsychotic

\section{Introduction}

Converging trends in the neuroimaging of schizophrenia have led to the research conducted in this study. The first trend is the association of altered caudate volume with antipsychotic treatment. Reduction in caudate volume has been reported in MRI studies of antipsychoticnaïve, first-episode schizophrenia patients (Corson et al. 1999a; Keshavan et al. 1998; Levitt et al. 2002; Shihabuddin et al. 1998), who should not exhibit effects associated with treatment and disease chronicity, however findings are inconsistent (Chakos et al. 1994; Gur et al. 1998a). Cross-sectional MRI studies suggest that treatment of schizophrenia with typical antipsychotic medications can be, but is not always, associated with enlarged caudate. Dose (Chakos et al. 1994) and treatment findings (Corson et al. 1999b; Keshavan et al. 1994; Lieberman et al. 2005; Massana et al. 2005) have been observed over periods of twelve to twenty-four months in longitudinal MRI studies. Treatment of schizophrenia with atypical antipsychotics for extended periods is associated with decreases in caudate volume (Corson et al. 1999b), although studies in rodents are not entirely consistent with the human data (Andersson et al. 2002; Lee et al. 1999).

The second trend is the reduction of volume (McClure and Weinberger, 2001) and deformation of shape (Csernansky et al. 1998, 2002) demonstrated in the hippocampus by cross-sectional MRI studies in schizophrenia. The size of the hippocampus is reduced at onset of the first episode of psychosis, before the effects associated with treatment and disease chronicity should occur (Lieberman et al. 2001; Szeszko et al. 2003; Velakoulis et al. 1999). Longitudinal MRI studies over treatment periods of two to two and a half years have shown hippocampal and temporal lobe volumes decrease (Gur et al. 1998b; Mathalon et al. 2001), though not all studies show such changes (Lieberman et al. 2001). The inconsistent findings should be viewed in the context of the common (Davis et al. 1998; Delisi et al. 1988, 1992, 1995, 1997; Gur et al. 1998b; Jacobsen et al. 1998; Lieberman et al. 2005; Rapport et al. 1997), but not ubiquitous (Garver et al. 2000; Lieberman et al. 2001) observation that regional grey and white matter volumes can decrease while CSF volumes increase in longitudinal schizophrenia studies. There have also been some reports of an association between hippocampal volume and antipsychotic type. In a cross-sectional MRI study, treatment with atypical antipsychotics was associated with larger hippocampal volumes than treatment with haloperidol, but the relationship was observed only in male patients early in the course of their illness (Chakos et al. 2002). In a longitudinal MRI study, first-episode schizophrenic subjects given haloperidol demonstrated more decreases in gray matter volume than those given olanzapine after 24, 52 and 104 weeks of treatment (Lieberman et al. 2005). While it is possible that antipsychotic type is associated with differences in hippocampal volume change, there is not conclusive evidence yet.

The third trend is the emergence of medial representations (M-reps); a shape analysis method providing information on a rich set of features not accessible by conventional volume-based morphometry (Fletcher et al. 2003; Han et al. 2005; Pizer et al. 2005). MRI studies performed at the UNC Neuro Image Research and Analysis Laboratory using these high-dimensional statistical descriptions of shape in lateral ventricles (Styner et al. 2003, 2005) and hippocampi (Styner et al. 2004), have demonstrated effects of genetic relatedness and schizophrenia, as well as a main effect of antipsychotic type in hippocampal shape of male schizophrenia patients (Chakos et al. 2002). 
This investigation examined caudate and hippocampal shape in schizophrenia patients randomly assigned to treatment with haloperidol or olanzapine. The specific purpose of the study was to determine if shape differences in the caudate and hippocampus of schizophrenia patients emerge following treatment. The specific outcome measures were two quantitative shape measurements: radius and position. These two shape measurements for the caudate and hippocampus were defined through M-reps with the radius measurement quantifying the local width at an M-rep node(represented in Figure 1 by the length of a thin rod projecting from a sphere) and the position measurement quantifying local deformation at an M-rep node (represented in Figure 1 by the location of a sphere).

The following questions were addressed: do haloperidol-, olanzapine-treated patients, and healthy control groups-globally (3-group comparison) or individually (comparisons between each group) - differ at baseline or in a repeated measures analysis (considering data from all time points but not longitudinal changes)? Over time, does shape change emerge globally among or individually between, the three groups? According to the a priori hypotheses, the following results were expected for shape measures in the hippocampus and caudate: (1) at baseline, an effect of diagnosis between schizophrenia patients and controls; (2) in the repeated measures analysis, an effect of diagnosis between schizophrenia patients and controls; (3) at baseline as well as in the repeated-measures analysis, no effect of antipsychotic type between schizophrenia patients in the two treatment groups; (4) in the global and individual group comparisons, differences will emerge over time.

\section{Materials and Methods}

Subjects were enrolled in a randomized, multi-site, double-blind study (Lieberman et al. 2005) that was conducted from March 1, 1997 to July 31, 2001 at 14 academic medical centers (11 in the United States, 1 in Canada, 1 in the Netherlands, and 1 in England).

In this study, 238 first-episode schizophrenia patients were enrolled. After random allocation at baseline, 123 patients were selected to receive the conventional antipsychotic haloperidol $(2-20 \mathrm{mg} / \mathrm{d})$, and 115 were selected to receive the atypical antipsychotic olanzapine (5-20 $\mathrm{mg} / \mathrm{d})$. Patients were treated and followed for up to 47 months. Fifty-six psychiatrically healthy subjects were matched to the patients' demographic characteristics and enrolled as control subjects (see Table 1 and Table 2). High resolution MRI (multi-site SPGR T1 weighted imaging on 1.5 Tesla scanners at $0.9375 \times 0.9375 \times 1.5 \mathrm{~mm}$ resolution) was performed at baseline and after 3, 6, and 12 months (Lieberman et al. 2005). A small set of scans were performed after 24 months which were not considered in this study. Standardized geometric phantoms were scanned twice per month to ensure geometric acuity and minimize scanner bias across the 14 medical centers.

Prior to structural segmentation, the baseline MRI scans were rigidly aligned (mutual information based registration) to a standard coordinate space and follow up MRI scans were globally, affinely aligned to their baseline data. This setup minimizes inherent effects due to changes over time in MR field-of-view (FOV) size or MR field inhomogeneity. In particular, we analyzed FOV variation across time by studying changes in scale-factors computed with affine co-registration of longitudinally paired geometric phantom acquisitions. These scale-factor measurements allowed us to estimate that within-site FOV size varied by as much as $3 \%$ over time, both within-slice-plane as well as out-of-plane, necessitating an affine alignment to correct for these FOV distortions. Not surprisingly these FOV variations varied across site, and thus affine alignment also reduces this site-specific bias. 
Caudate and hippocampus structures were then segmented from the aligned MRI scans with an automated atlas based segmentation tool developed in-house called AutoSeg (Gouttard et al. 2007). Caudate and hippocampus shape were next represented as a medial mesh of sampled nodes via a statistically-constrained based fit of a prior medial mesh model into the binary segmentations (Pizer et al. 2009; Styner et al. 2003).

Figure 1 illustrates the medial mesh for both structures with the associated medial node labeling used in the result section. Shape was captured as local width (medial width/radius) and local deformation (medial deformation) as proposed by Carracso et al. (2000). While local size is independent of pose, appropriate local deformation analysis necessitates a prior alignment. Alignment was performed for the caudate and hippocampus independently, via an extension of the standard rigid Procrustes alignment to medial structures (Gorczowski et al. 2010).

The aim of our study was to investigate differences in medial width and deformation of the hippocampus and caudate across the three groups (haloperidol-treated, olanzapine-treated, and controls) while controlling for other covariates of interest. We utilized a novel nonparametric method called adjusted exponentially tilted (ET) likelihood, along with a likelihood ratio test for hypothesis testing (Zhu et al. 2009). Assuming only a set of estimating equations, the adjusted ET likelihood is a nonparametric extension of general linear mixed models and generalized estimating equations. This extension of the ET method as a nonparametric method is particularly desirable for the analysis of brain morphometry because the distribution of the morphometric measures often deviates from the Gaussian distribution. For this study, the Shapiro-Wilk normality test was applied to check this parametric assumption at each atom for both left and right hippocampi and caudate using the residuals. It turned out that the Shapiro-Wilk test rejected the normality assumption at many atoms of both structures on each side; therefore our nonparametric adjusted ET method is preferred for the analysis of this data.

We first tested the a priori hypothesis at baseline. We used the adjusted ET likelihood to calculate regression parameter estimates and then tested for group differences (Zhu et al. 2009). Specifically, at each atom, we considered the moment model based on the $28 \times 1$ estimating function. The dependent variables were local width and local deformation, determined at 24 hippocampal nodes and 21 caudate nodes using M-reps (see Figure 1). Covariates of interest were WBV (whole brain volume $=$ CSF, white matter and gray matter), ethnicity, age, gender, group (the two schizophrenia groups and the healthy control group), and time. The adjusted ET likelihood ratio statistic is used to test hypothesis of interest. Please see Zhu et al. (2009) for detailed analysis.

The false discovery rate approach was then used to correct for multiple comparisons in all atoms for each structure (Benjamini and Hochberg, 1995). Secondly, we performed longitudinal data analyses (Liang and Zeger, 1986). Again, we used the adjusted ET likelihood coupled with the generalized estimating equations for longitudinal data to estimate the regression parameters and then tested for specific group differences. In the longitudinal analysis, we used the similar set of estimating equations as those in the baseline analysis. Based on our previous analysis in Shi et al. (2011), we treated WBV as type II covariate and adjusted the estimating equations slightly. Please see Shi et al. (2011) for detailed analysis. 


\section{Results}

\subsection{Hippocampal Shape: Local Width and Local Deformation(See Fig 2)}

The longitudinal analysis did not reveal significant changes in either local width or deformation in the hippocampus (see Tables 3 and 4). The longitudinal global comparison demonstrated a Group $\times$ Time interaction of width at nodes M8, C2, C3, and L6 in the left hippocampus (see Figure 1), but only marginally reached statistical significance (Table 3). Longitudinal analysis of the individual groups demonstrated no effect of time for local width in any region of the left hippocampus (Table 3). Neither the global nor the individual group comparisons, demonstrated an interaction of Group $\times$ Time or an effect of Time with respect to local deformation of the left hippocampus longitudinally (see Table 4). Longitudinal analysis also failed to show any Group $\times$ Time interaction or an effect of Time with respect to local width or deformation in the right hippocampus (see Tables 3 and 4).

At baseline and in repeated measures analysis (using measurements from all the time points), patients demonstrated significantly reduced node width (meaning a significantly reduced local volume near the node location) when compared to controls (see Tables 5, 6, 7, and 8). Significant baseline differences in width were observed in comparison of the haloperidol-treated patients and controls, localized to node M8, C3, C4, and C5 in the left hippocampus. The repeated measures analysis of haloperidol-treated patients compared to controls revealed significant differences in width at nodes M8, C3 and C4 in the left hippocampus. Significant differences in width were observed in the baseline analysis comparison of olanzapine-treated patients to controls, located at nodes C4 and C5 of the left hippocampus. Repeated-measures analysis of olanzapine-treated patients compared to controls did not show significant differences in width at any left or right hippocampal region. As expected, neither the baseline nor the repeated-measures analysis showed any significant differences in width between haloperidol and olanzapine-treated patients for the left hippocampus.

In both the baseline and the repeated-measures analysis, each patient group showed local differences in hippocampal deformation at the medial nodes compared to controls (see Tables 7 and 8). Haloperidol-treated patients compared to controls, showed differences in local deformation throughout medial, central, and lateral regions of the left hippocampus in both the baseline and repeated-measures analyses. In the baseline analysis only, the olanzapine-treated patients showed differences from controls in local deformation in medial, central, and lateral nodes for the left hippocampus, overlapping with the nodes showing differences in the comparison of haloperidol-treated patients to controls. However in the repeated-measures analysis, olanzapine-treated patients compared to controls showed differences in local deformation in only a few left hippocampal nodes (M8, C1, and L4).

No significant differences in local deformation of the left hippocampus were observed between haloperidol- or olanzapine-treated patients in the baseline or repeated-measures analyses (Tables 7 and 8). In the right hippocampus, differences in local deformation were observed in the repeated measures analysis between haloperidol-treated patients and controls at nodes M1, M7, M8, C1, C3, C6, C7, L2, and L7, but no differences in local deformation were noted in the right hippocampus between either the olanzapine-treated patients and controls or the olanzapine and haloperidol-treated patients.

\subsection{Caudate Shape: Local Width and Local Deformation (See Fig 3)}

There was little evidence for significant longitudinal caudate shape change in local width or deformation (see Tables 9 and 10). Global comparison of the three groups in the longitudinal analysis demonstrated a Group $\times$ Time interaction in local width of the left caudate at nodes S5 and I1 (see Fig 1), but these findings only marginally reached statistical significance. No 
effect of Time was observed in local width at any node in the individual group analysis. In the right caudate, the longitudinal global comparison demonstrated a Group $\times$ Time interaction in local width at nodes S1, S4 and I2, but again no effect of Time was observed in the individual group analysis. Longitudinal analysis of deformation did not show significant effects in either left or right caudate and no Group $\times$ Time interaction was observed.

The baseline and repeated measures analyses of left and right caudate width showed highly similar results with respect to regional specificity (Tables 11, 12). While no left caudate width differences were observed between any groups, haloperidol-treated patients compared to controls showed significant differences in right caudate width for nodes S4 and I7 at baseline. The repeated measures analysis also showed right caudate width differences at node I7. Similarly, olanzapine-treated patients compared to controls had significant right caudate width differences at node $\mathrm{S} 4$ at baseline, but not in the repeated measures analysis. No significant differences in the right caudate were observed between treatment groups.

The repeated measures analyses of local deformation in patient groups compared to controls showed widespread differences in the caudate (Tables 13 and 14). In the baseline analysis, haloperidol-treated patients showed differences from controls in deformation of the left caudate at nodes S2-8, C1, C3, C4, and I1-I6, which was also reflected in the repeated measures analysis where they showed significant differences in deformation of nodes S2, S5, S6, C3, I1, I2 and I6. Compared to controls, olanzapine-treated patients displayed differences in deformation in left caudate nodes S2-5, C1, C4, I1-4, I6, and I7. One superior node in the left caudate (S2) showed differences in deformation when the haloperidoltreated and the olanzapine-treated patients were compared (Table 14), but this finding was regionally isolated and no difference in width was observed. Repeated measures analysis of right caudate deformation demonstrated similar differences between treatment groups and controls. Differences in local deformation were observed in haloperidol-treated patients at nodes S2-6, C1, C5, I1-6 and in olanzapine-treated patients at nodes S1-5, C1 and C2, C5, I1-I4, I6 and I7. No differences in deformation were noted in the right caudate between treatment groups (Table 14).

\section{Discussion}

Longitudinal changes in local width and deformation were not observed globally or when the haloperidol-treated, olanzapine-treated, and control groups were examined individually. While no differences were found between the treatment groups for the caudate or hippocampus, both treatment groups showed significant differences from controls. The baseline analysis demonstrated differences in local width of the left hippocampus at centralmiddle areas between both treatment groups and controls with the repeated measures analysis showing the same result for only the haloperidol-treated patients. Differences in local deformation between each treatment group and controls were observed at baseline in the left hippocampus at anterior, middle, and posterior nodes. Furthermore, at baseline haloperidol-treated patients and controls also showed differences in local deformation for anterior and posterior nodes of the right hippocampus. These baseline hippocampal shape differences are expected as previous studies have found differential hippocampal volumes for first episode schizophrenic patients (Lieberman et al. 2001; Velakoulis et al. 1999) with Szeszko et al. (2003) specifically finding reduced volume for the anterior hippocampus. A previous study by Styner et al. (2004) in chronic schizophrenia patients reported significant deformation differences in a few posterior (tail) nodes after normalization for hippocampal size differences. 
Baseline and repeated measures analyses demonstrated differences in local deformation between patients and controls at several superior and inferior nodes of both the right and left caudate, with considerable regional overlap between the treatment groups. These broad deformation differences were expected as other studies have shown similar differences to be present in anti-psychotic naïve schizophrenics (Ballmaier et al. 2008) and even in the unaffected siblings of schizophrenia patients (Mamah et al. 2008). Local width differences between patients and controls were seen at baseline only for the right caudate and only in isolated superior (S4) and head (I7) regions. This finding is particularly consistent with those of Levitt et al (2009) which showed a very similar pattern of significant isolated local shape differences for males with schizotypal personality disorder that appeared only in the right caudate using a surface based shape analysis. While such a surface based analysis can provide a higher degree of locality, it cannot separate changes in local width (growth or shrinkage) from changes in local deformation as our M-Rep method can. See Styner et al. (2004) for a more detailed comparison of these two methods of shape analysis.

Surprisingly, significant differences in shape change were not observed over time between haloperidol- and olanzapine-treated patients. There are several potential explanations for why our primary hypothesis was not supported. First, the results could be false-negative, and while significant shape differences did emerge between the treatment groups, our methods did not detect them. This is possible but unlikely, given that shape differences were observed at baseline between controls and schizophrenics, with considerable regional overlap between the two treatment groups. Second, it is possible our results are true negative. The structural changes in the caudate associated with antipsychotic treatment are small in size and not seen in every study (Mamah et al. 2007; Tauscher-Wisniewski et al. 2002). Third, although shape measures are thought to be more sensitive than volume measures, the shape change associated with antipsychotic treatment may be smaller than volume changes, and undetectable using the M-reps method. Fourth, unidentified confounding factors may have masked the effects of antipsychotic treatment. Confounding factors certainly could have masked actual shape change, since it is not possible to match subjects on every factor that may alter brain structure. Several factors may have altered the brain structures measured with MRI in this population: disease progression (McClure and Lieberman, 2003); external environmental events associated with schizophrenia such as changes in tissue perfusion, fat content, and water content; changes in the neuronal and nonneuronal tissue compartments in the brain (Weinberger and McClure, 2002). Other potentially confounding factors not controlled for in this study include hydration status, body weight, illness severity, and previous treatment with antipsychotic medication.

This study is the first large-scale analysis that specifically examines longitudinal changes in local width and deformation in schizophrenia patients treated with typical and atypical antipsychotics using the M-reps method. The absence of shape differences at baseline between schizophrenia patients randomized into treatment groups indicates that our execution of the M-reps method was stable and reliable. Differences in the shape of the hippocampus and caudate between schizophrenic patients and controls using these methods is a novel finding, though not unexpected. There is extensive evidence of anatomical specificity in connections between the basal ganglia, hippocampus, and cerebral cortex (Draganski et al. 2008). There is also evidence from functional neuroimaging studies suggesting the presence of dysfunctional prefrontal-hippocampal (Callicott et al. 2003) and prefrontal-basal ganglia (Morey et al. 2008) circuits in schizophrenia. Demonstrating a direct relationship between dysfunction of specific circuits and altered morphometry of the caudate and hippocampus would provide additional evidence for the disruption of those circuits in schizophrenia. 


\section{Acknowledgments}

Work supported by the Stanley Medical Research Institute, UNC Neurodevelopmental Disorders Research Center HD 03110, NIH NIBIB grant P01 EB002779, Eli Lilly user initiated information technology grant PCG TR: 033107/F1D-MC-X252, NIH grants UL1-RR025747-01, MH086633, P01CA142538-01, AG033387 and the NIH Roadmap Grant U54 EB005149-01, National Alliance for Medical Image Computing.

\section{References}

Andersson C, Hamer RM, Lawler CP, Mailman RB, Lieberman JA. Striatal volume changes in the rat following long-term administration of typical and atypical antipsychotic drugs.

Neuropsychopharmacology. 2002; 27(2):143-151. [PubMed: 12093588]

Ballmaier M, Schlagenhauf F, Toga AW, Gallinat J, Koslowski M, Zoli M, Hojatkashani C, Narr KL, Heinz A. Regional patterns and clinical correlates of basal ganglia morphology in non-medicated schizophrenia. Schizophrenia Research. 2008; 106(2-3):140-147. [PubMed: 18818054]

Benjamini Y, Hochberg Y. Controlling the false discovery rate: a practical and powerful approach to multiple testing. Journal of the Royal Statistical Society, Series B (Methodological). 1995; 57(1): 289-300.

Callicott JH, et al. Complexity of prefrontal cortical dysfunction in schizophrenia: more than up or down. American Journal of Psychiatry. 2003; 160(12):2209-2215. [PubMed: 14638592]

Carracso J, Penkowa M, Hadberg H, Molinero A, Hidalgo J. Enhanced seizures and hippocampal neurodegeneration following kainic acid-induced seizure in metallothionein-I + II-deficient mice. European Journal of Neuroscience. 2000; 12:2311-2322. [PubMed: 10947810]

Chakos M, Lieberman JA, Bilder RM, Borenstein M, Lerner G, Bogerts B, Wu H, Kinon B, Manzar A. Increase in Caudate Nuclei Volumes of First-Episode Schizophrenic Patients Taking Antipsychotic Drugs. American Journal of Psychiatry. 1994; 151:1430-1436. [PubMed: 7916539]

Chakos MH, Schobel S, Gu H, Gerig G, Bradford D, Charles C, Lieberman J. Duration of illness and treatment effects on hippocampal volume in male patients with schizophrenia. British Journal of Psychiatry. 2005

Corson P, Nopoulosa P, Andreasen NC, Heckela D, Arndta S. Caudate size in first-episode neuroleptic-naive schizophrenic patients measured using an artificial neural network. Biological Psychiatry. 1999a; 46(5):712-720. [PubMed: 10472424]

Corson PW, Nopoulos P, Miller DD, Arndt S, Andreason NC. Change in Basal Ganglia Volume Over 2 Years in Patients with Schizophrenia: Typical Versus Atypical Neuroleptics. American Journal of Psychiatry. 1999b; 156(8):1200-1204. [PubMed: 10450260]

Csernansky JG, Joshi S, Wang L, Haller JW, Gado M, Miller JP, Grenander U, Miller MI. Hippocampal morphometry in schizophrenia by high dimensional brain mapping. Proceedings of the National Academy of Sciences of the United States of America. 1998; 95(19):11406-11411. [PubMed: 9736749]

Csernansky JG, Wang L, Jones D, Rastogi-Cruz D, Posener JA, Heydebrand G, Miller JP, Miller MI. American Journal of Psychiatry. 2002; 159(12):2000-2006. [PubMed: 12450948]

Davis K, Buchsbaum MS, Shihabuddin L, Speigel-Cohen J, Metzger M, Frecska E, Keefe RS, Powchick P. Ventricular enlargement in poor-outcome schizophrenia. Biological Psychiatry. 1998; 43:783-793. [PubMed: 9611667]

DeLisi L, Dauphinas ID, Gershon ES. Perinatal complications and reduced size of brain limbic structures in familial schizophrenia. Schizophrenia Bulletin. 1988; 14:185-191. [PubMed: 3201176]

DeLisi L, Sakuma M, Tew W, Kushner M, Hoff AL, Grimson R. Schizophrenia as a chronic active brain process: a study of progressive brain structural change subsequent to the onset of schizophrenia. Psychiatry Research: Neuroimaging. 1997; 74:129-140.

DeLisi L, Tew W, Xie S, Hoff AL, Sakuma M, Kushner M, Lee G, Shedlack K, Smith AM, Grimson R. A prospective follow-up study of brain morphology and cognition in first-episode schizophrenic patients: preliminary findings. Biological Psychiatry. 1995; 38:349-360. [PubMed: 8547454] 
DeLisi LE, et al. The timing of brain morphological changes in schizophrenia and their relationship to clinical outcome. Biological Psychiatry. 1992; 31(3):241-254. [PubMed: 1547298]

Draganski B, Kherif F, Kloppel S, Alexander C, Parker GJ, Deichmann R, Ashburner J, Frackowiak RS. Evidence for segregated and integrative connectivity patterns in the human Basal Ganglia. Journal of Neuroscience. 2008; 28(28):7143-7152. [PubMed: 18614684]

Fletcher P, Joshi S, Lu C, Pizer S. Gaussian distributions on Lie groups and their application to statistical shape analysis. Information Processing in Medical Imaging. 2003; 18:450-462. [PubMed: 15344479]

Garver D, Nair TR, Chrisensen JD, Holcomb JA, Kingsbury SL. Brain and ventricular instability during psychotic episodes of the schizophrenias. Schizophrenia Research. 2000; 44:11-23. [PubMed: 10867308]

Gogtay N, et al. Comparison of progressive cortical gray matter loss in childhood-onset schizophrenia with that in childhood-onset atypical psychoses. Archives of General Psychiatry. 2004; 61(1):1722. [PubMed: 14706940]

Gorczowski K, Styner M, Jeong JY, Marron JS, Piven J, Hazlett HC, Pizer SM, Gerig G. Multi-object analysis of volume, pose, and shape using statistical discrimination. IEEE Transactions on Pattern Analysis and Machine Intelligence. 2010; 32(4):652-661. [PubMed: 20224121]

Gouttard S, Styner M, Joshi S, Gerig G. Subcortical structure segmentation using probabilistic atlas prior. Proceedings of SPIE Medical Imaging. 2007; 65122:J1-J11.

Gur R, Cowell P, Turetsky BI, Gallacher F, Cannon T, Bilker W, Gur RC. A follow-up magnetic resonance imaging study of schizophrenia. Archives of General Psychiatry. 1998b; 55:145-152. [PubMed: 9477928]

Gur R, Maany V, Mozley PD, et al. Subcortical MRI volumes in neuroleptic naive and treated patients with schizophrenia. American Journal of Psychiatry. 1998a; 155:1711-1717. [PubMed: 9842780]

Han Q, Pizer SM, Merck D, Joshi S, Jeong JY. Multi-figure anatomical objects for shape statistics. Information Processing in Medical Imaging. 2005; 19:701-712. [PubMed: 17354737]

Jacobsen L, Giedd JN, Castellanos FX, Vaituzis AC, Hamburger SD, Kumra S, Lenane MC, Rapoport JL. Progressive reduction of temporal lobe structures in childhood-onset schizophrenia. American Journal of Psychiatry. 1998; 155:678-685. [PubMed: 9585721]

Keshavan M, Bagwell WW, Haas GL, Sweeney JA, Schooler NR, Pettegrew JW. Changes in caudate volume with neuroleptic treatment. The Lancet. 1994; 344:1434.

Keshavan MS, Rosenberg D, Sweeney JA, Pettegrew JW. Decreased caudate volume in neurolepticnaive psychotic patients. American Journal of Psychiatry. 1998; 155(6):774-778. [PubMed: 9619149]

Lee $\mathrm{H}$, et al. Effects of chronic treatment with typical and atypical antipsychotic drugs on the rat striatum. Life Sciences. 1999; 64(18):1595-1602. [PubMed: 10328519]

Levitt JJ, McCarley RW, Dickey CC, Voglmaier MM, Niznikiewicz MA, Seidman LJ, Hirayasu Y, Ciszewski AA, Kikinis R, Jolesz FA, Shenton ME. MRI Study of Caudate Nucleus Volume and Its Cognitive Correlates in Neuroleptic-Naive Patients With Schizotypal Personality Disorder. American Journal of Psychiatry. 2002; 159(7):1190-1197. [PubMed: 12091198]

Levitt JJ, Steiner M, Niethammer M, Sylvain B, Koo MS, Voglmaier MM, Dickey CC, Niznikiewicz MA, Kikinis R, Robert WM, Shenton ME. Shape abnormalities of caudate nucleus in schizotypal personality disorder. Schizophrenia Research. 2009; 110(1-3):127-139. [PubMed: 19328654]

Liang K, Zeger SL. Longitudinal data analysis using generalized linear models. Biometrika. 1986; 73:13-22.

Lieberman J, Chakos MA, Wu H, Alvir J, Hoffman E, Robinson D, Bilder R. Longitudinal study of brain morphology in first episode schizophrenia. Biological Psychiatrysource. 2001; 49:487-499.

Lieberman JA, Tollefson GD, Charles C, Zipursky R, Sharma T, Kahn RS, Keefe RSE, Green AI, Gur RE, McEvoy J, Perkins D, Hamer RM, Gu H, Tohen M. HGDH Study Group. Antipsychotic drug effects on brain morphology in first-episode psychosis. Archives of General Psychiatry. 2005; 62(4):361-370. [PubMed: 15809403]

Mamah D, Harms MP, Wang L, Barch D, Thompson P, Kim J, Miller MI, Csernansky JG. Basal ganglia shape abnormalities in the unaffected siblings of schizophrenia patients. Biological Psychiatry. 2008; 64(2):111-120. [PubMed: 18295189] 
Mamah D, Wang L, Barch D, de Erausquin GA, Gado M, Csernansky JG. Structural analysis of the basal ganglia in schizophrenia. Schizophrenia Research. 2007; 89(1-3):59-71. [PubMed: 17071057]

Massana G, et al. Volume changes in gray matter in first-episode neuroleptic-naive schizophrenic patients treated with risperidone. Journal of Clinical Psychopharmacology. 2005; 25(2):111-117. [PubMed: 15738741]

Mathalon D, Sullivan EV, Lim KO, Pfefferbaum A. Progressive brain volume changes and the clinical course of schizophrenia in men. Archives of General Psychiatry. 2001; 58:148-157. [PubMed: 11177116]

McClure RK, Lieberman JA. Neurodevelopmental and neurodegenerative hypothesise of schizophrenia: a review and critique. Current Opinion in Psychiatry. 2003; 16(Supplement 2):S15S28.

McClure, RK.; Weinberger, DR. The Neurodevelopmental Hypothesis of Schizophrenia: A Review of the Evidence. In: Breier, A.; Tran, PV.; Herrea, HM.; Tollefson, GD.; Bymaster, FP., editors. Current Issues in the Psychopharmacology of Schizophrenia. Philadelphia: Lippincott William and Wilkins Healthcare; 2001. p. 27-56.

Morey R, Mitchell TV, Inan S, Lieberman JA, Belger A. Neural correlates of automatic and controlled auditory processing in schizophrenia. Journal of Neuropsychiatry and Clinical Neurosciences. 2008; 20(4):419-430. [PubMed: 19196926]

Pizer S, Fletcher PT, Joshi S, Gash AG, Stough J, Thall A, Tracton G, Chaney EL. A method and software for segmentation of anatomic object ensembles by deformable m-reps. Medical Physics. 2005; 32(5):1335-1345. [PubMed: 15984685]

Rapoport JL, et al. Childhood-onset schizophrenia: progressive ventricular change during adolescence. Archives of General Psychiatry. 1997; 54:897-903. [PubMed: 9337768]

Shi XY, Ibrahim JG, Styner M, Yimei L, Zhu HT. Two-stage adjusted exponential tilted empirical likelihood for neuroimaging data. Annals of Applied Statistics. 2011; 5:1132-1158. [PubMed: 21765885]

Shihabuddin L, Buchsbaum MS, Hazlett EA, Haznedar M, Harvey PD, Newman A, Schnur DB, Spiegel-Cohen J, Tsechung W, Machac J, Knesaurek K, Vallabhajosula S, Biren MA, Ciaravolo TM, Luu-Hsia C. Dorsal Striatal Size, Shape, and Metabolic Rate in Never-Medicated and Previously Medicated Schizophrenics Performing a Verbal Learning Task. Archives of General Psychiatry. 1998; 55:235-243. [PubMed: 9510217]

Styner M, Gerig G, Lieberman J, Jones D, Weinberger D. Statistical shape analysis of neuroanatomical structures based on medial models. Medical Image Analysis. 2003; 7(3):207-220. [PubMed: 12946464]

Styner M, Lieberman JA, McClure RK, Weinberger DR, Jones DW, Gerig G. Morphometric analysis of lateral ventricles in schizophrenia and healthy controls regarding genetic and disease-specific factors. Proceedings of the National Academy of Sciences of the United States of America. 2005; 102(13):4872-4877. [PubMed: 15772166]

Styner M, Lieberman JA, Pantazis D, Gerig G. Boundary and medial shape analysis of the hippocampus in schizophrenia. Medical Image Analysis. 2004; 8(3):197-203. [PubMed: 15450215]

Szeszko PR, Goldberg E, Gunduz-Bruce H, Ashtari M, Robinson D, Malhotra AK, Lencz T, Bates J, Crandall DT, Kane JM, Bilder RM. Smaller anterior hippocampal formation volume in antipsychotic-naive patients with first-episode schizophrenia. American Journal of Psychiatry. 2003; 160(12):2190-2197. [PubMed: 14638589]

Tauscher-Wisniewski S, Tauscher J, Logan J, Christensen BK, Mikulis DJ, Zipursky RB. Caudate volume changes in first episode psychosis parallel the effects of normal aging: a 5-year follow-up study. Schizophrenia research. 2002; 58(2-3):185-188. [PubMed: 12409157]

Velakoulis D, et al. Hippocampal volume in first-episode psychoses and chronic schizophrenia: a highresolution magnetic resonance imaging study. Archives of General Psychiatry. 1999; 56(2):133141. [PubMed: 10025437] 
Weinberger DR, McClure RK. Neurotoxicity, neuroplasticity, and magnetic resonance imaging morphometry: what is happening in the schizophrenic brain? Archives of General Psychiatry. 2002; 59(6):553-558. [PubMed: 12044198]

Zhu H, Zhou H, Chen J, Li Y, Lieberman J, Styner M. Adjusted Exponentially Tilted Likelihood with Applications to Brain Morphology. Biometrics. 2009; 65:919-927. [PubMed: 18945269] 

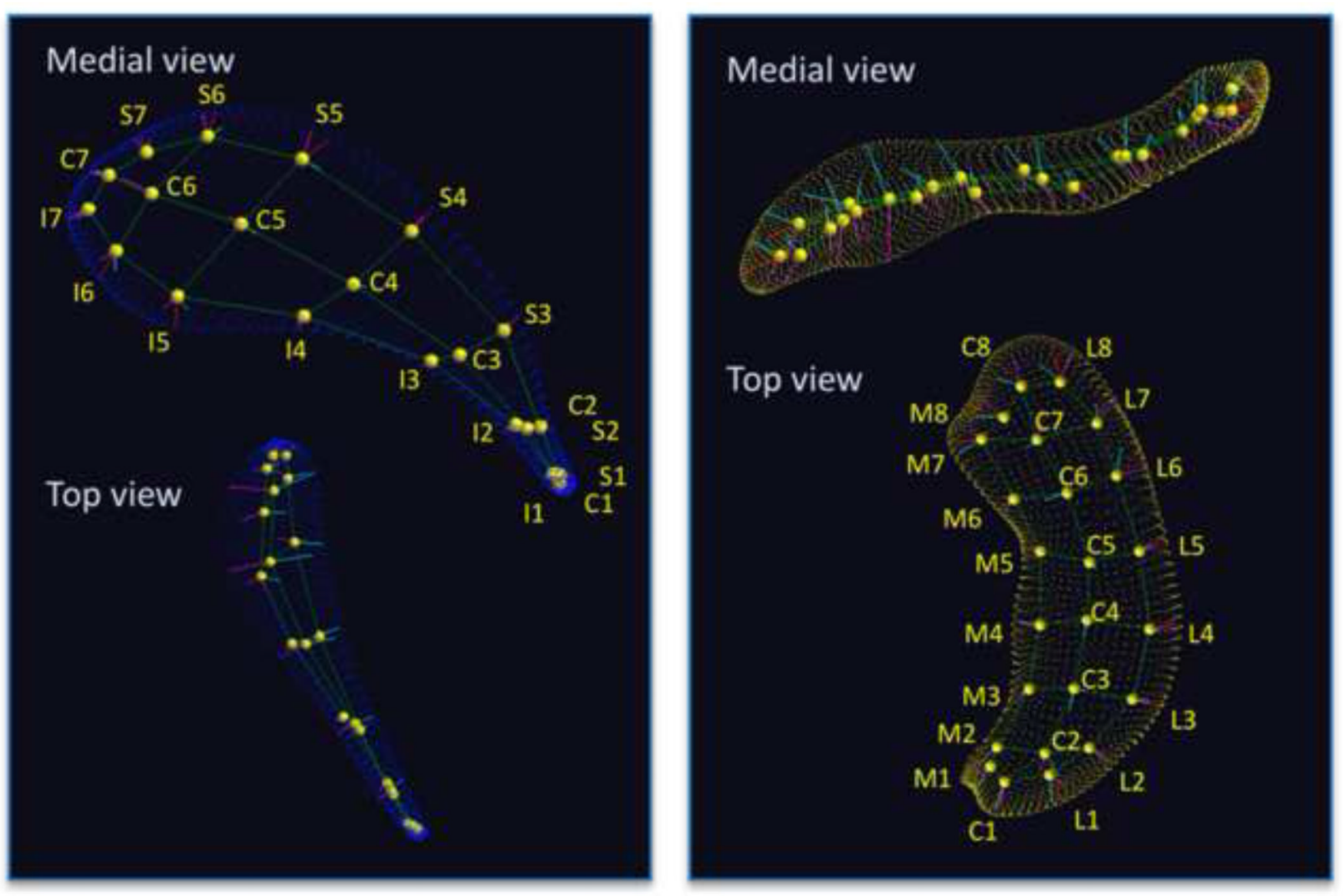

Figure 1.

Visualization of the medial representation grid and node labeling for right caudate (left, labeled from a medial view) and hippocampus (right, labeled from the top view). Labels: $\mathrm{S}=$ Superior, $\mathrm{C}=$ Central, $\mathrm{I}=$ Inferior, $\mathrm{M}=$ Medial, $\mathrm{L}=$ Lateral. Increasing numerical value means going from a posterior to an anterior location. Local radius is represented by the length of the thin rods projecting from the node points, the node width. Local position is represented by the location of the node points measuring local deformation. 


\section{Hippocampus}

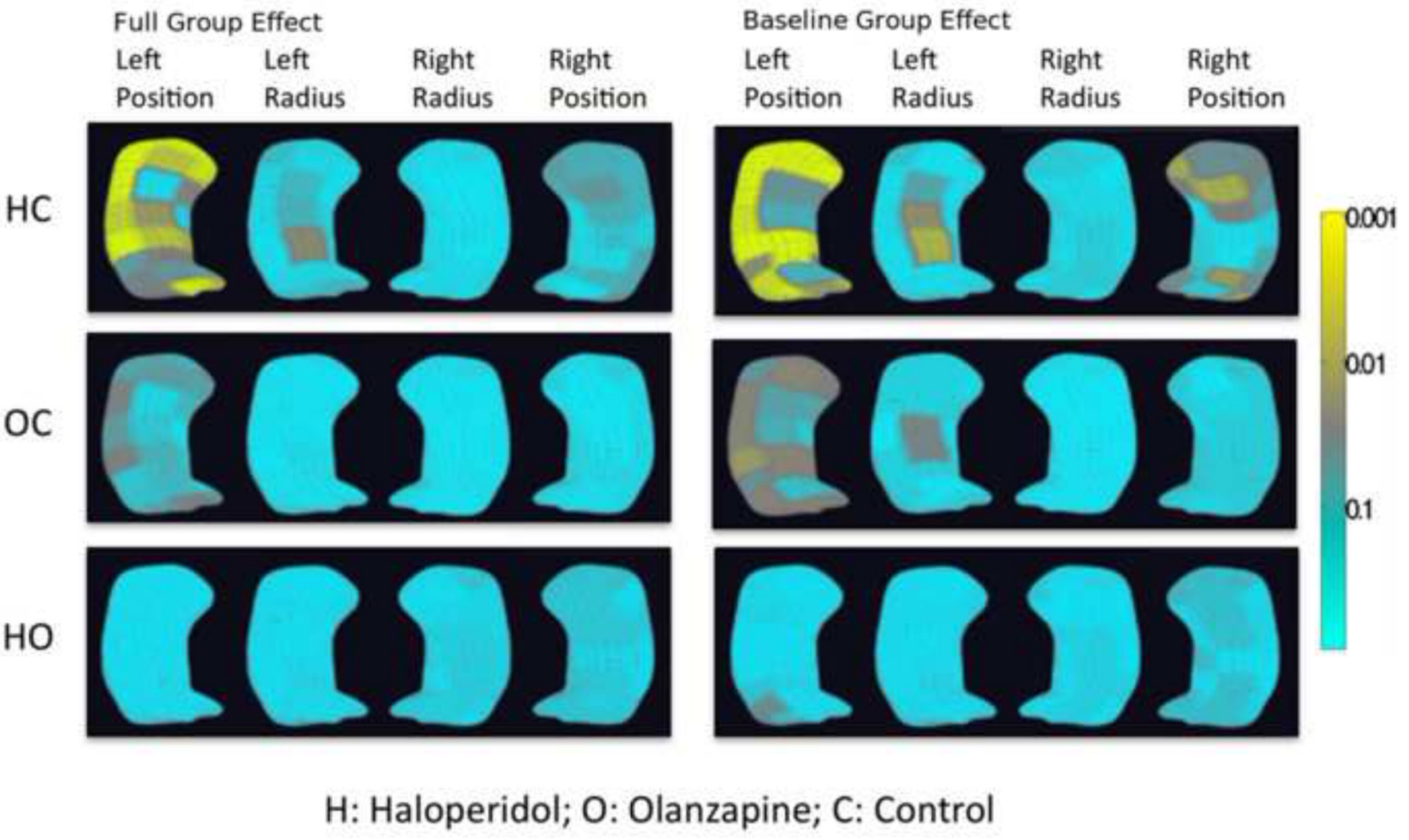

Figure 2.

Visualization of local significance in medial position and width in the hippocampus. Left: Baseline group effect only (without repeated measures), Right: Full group effect. P-values are visualized from cyan $(\mathrm{p}=0.1)$ to yellow $(\mathrm{p}=0.001)$. All maps are corrected for multiple comparisons. 


\section{Caudate}

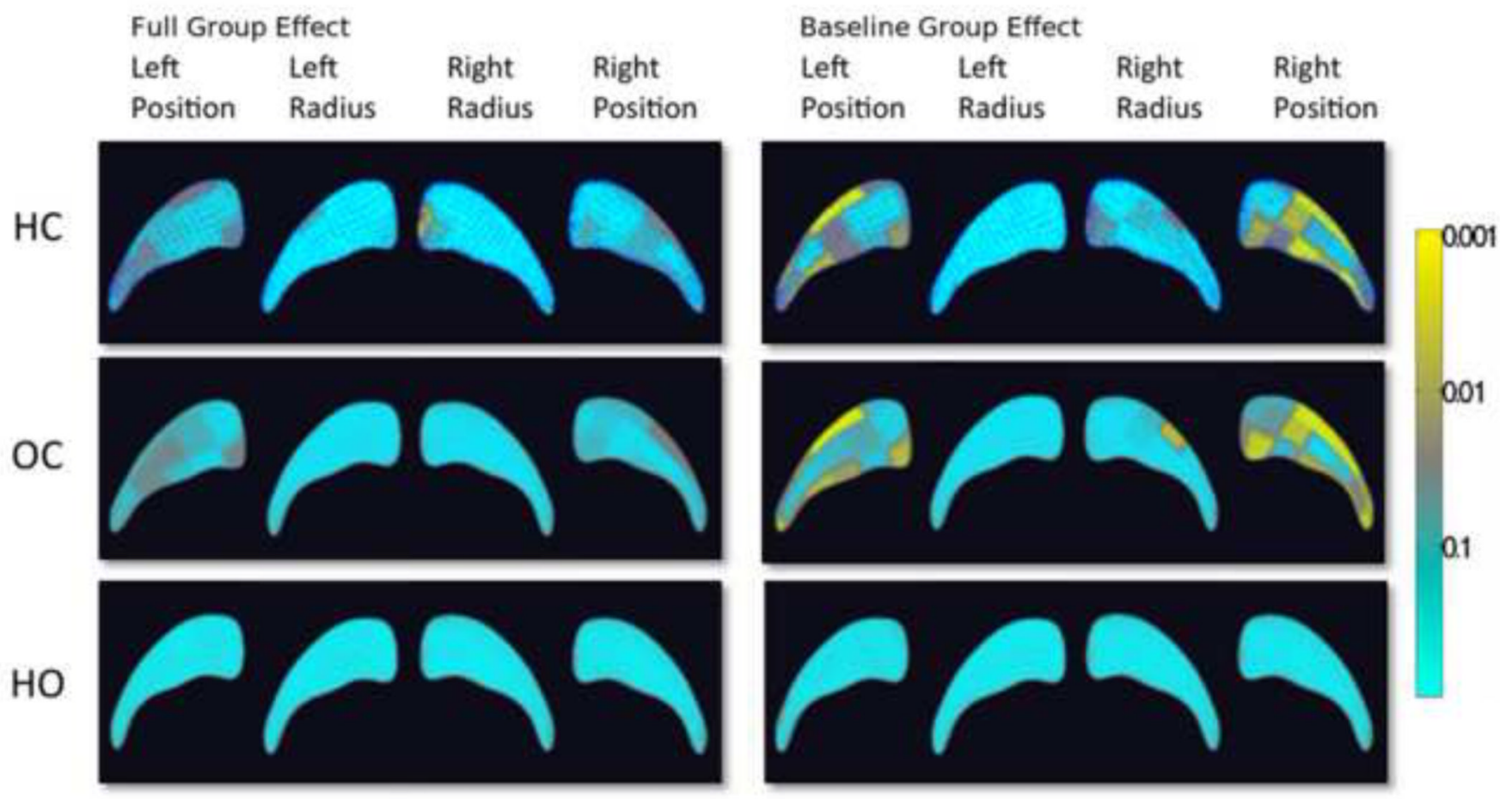

\section{H: Haloperidol; O: Olanzapine; C: Control}

Figure 3.

Visualization of local significance in medial position and radius in the caudate. Left: Baseline group effect only (without repeated measures), Right: Full group effect. P-values are visualized from cyan $(\mathrm{p}=0.1)$ yellow $(\mathrm{p}=0.001)$. All maps are corrected for multiple comparisons. 


\section{Table 1}

Demographic Characteristics of Patients and Controls at Baseline

\begin{tabular}{lrrcr}
\hline & \multicolumn{2}{c}{ Age (years) } & $\begin{array}{c}\text { Baseline Whole Brain Volume } \\
\text { (liters) }\end{array}$ \\
\cline { 2 - 5 } & Mean & SS.D. & Mean & SS.D. \\
\hline Haloperidol & 24.14 & 4.89 & 1.335 & 0.129 \\
\hline Olanzapine & 23.56 & 4.63 & 1.330 & 0.143 \\
\hline Controls & 25.28 & 3.97 & 1.368 & 0.127 \\
\hline
\end{tabular}




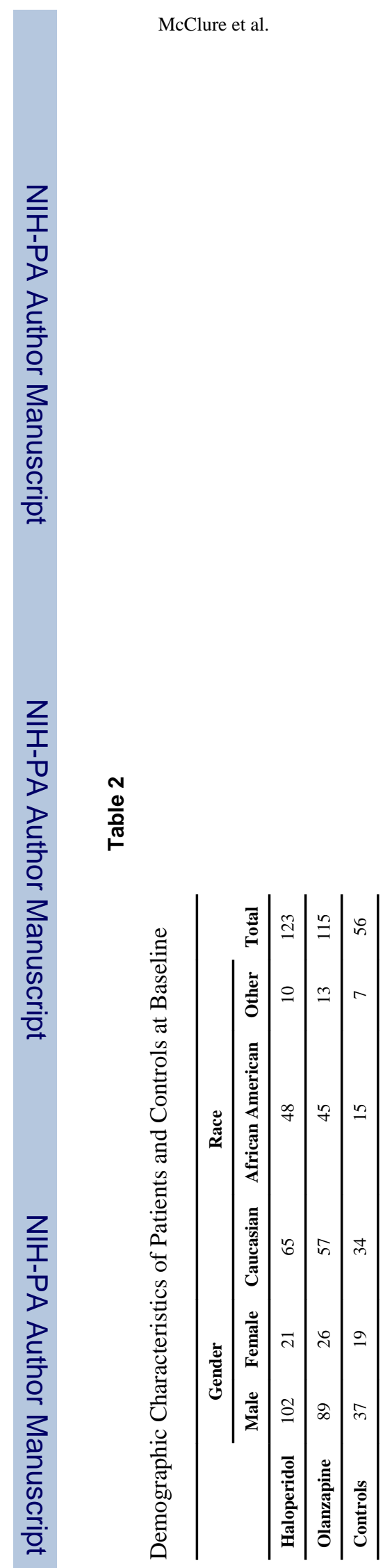

Psychiatry Res. Author manuscript; available in PMC 2014 January 30. 


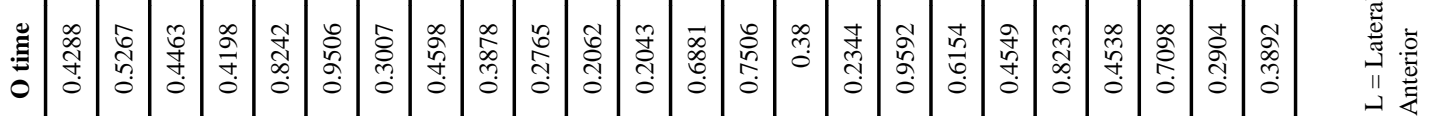

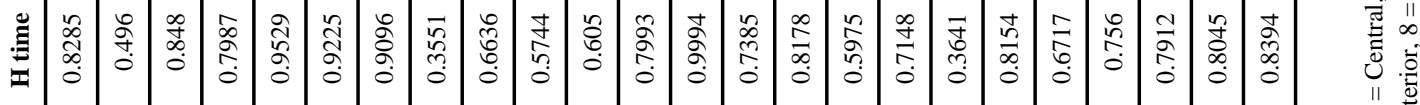

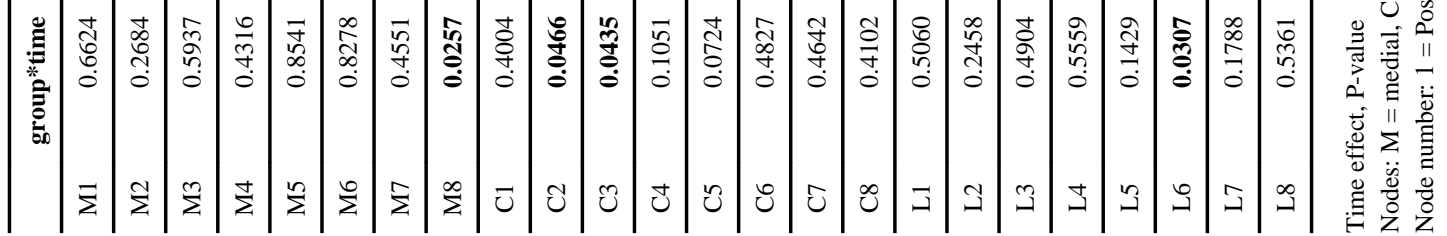




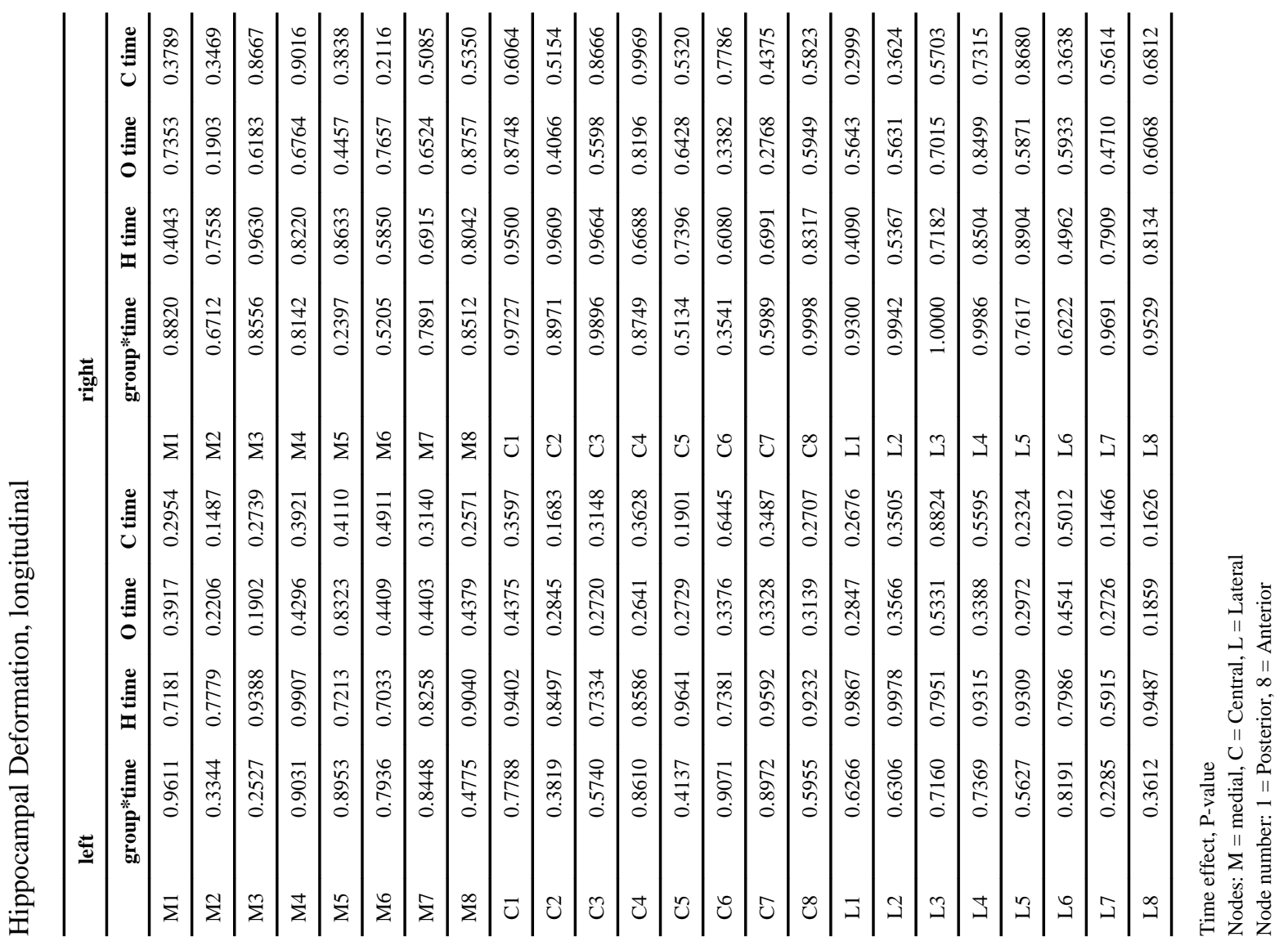




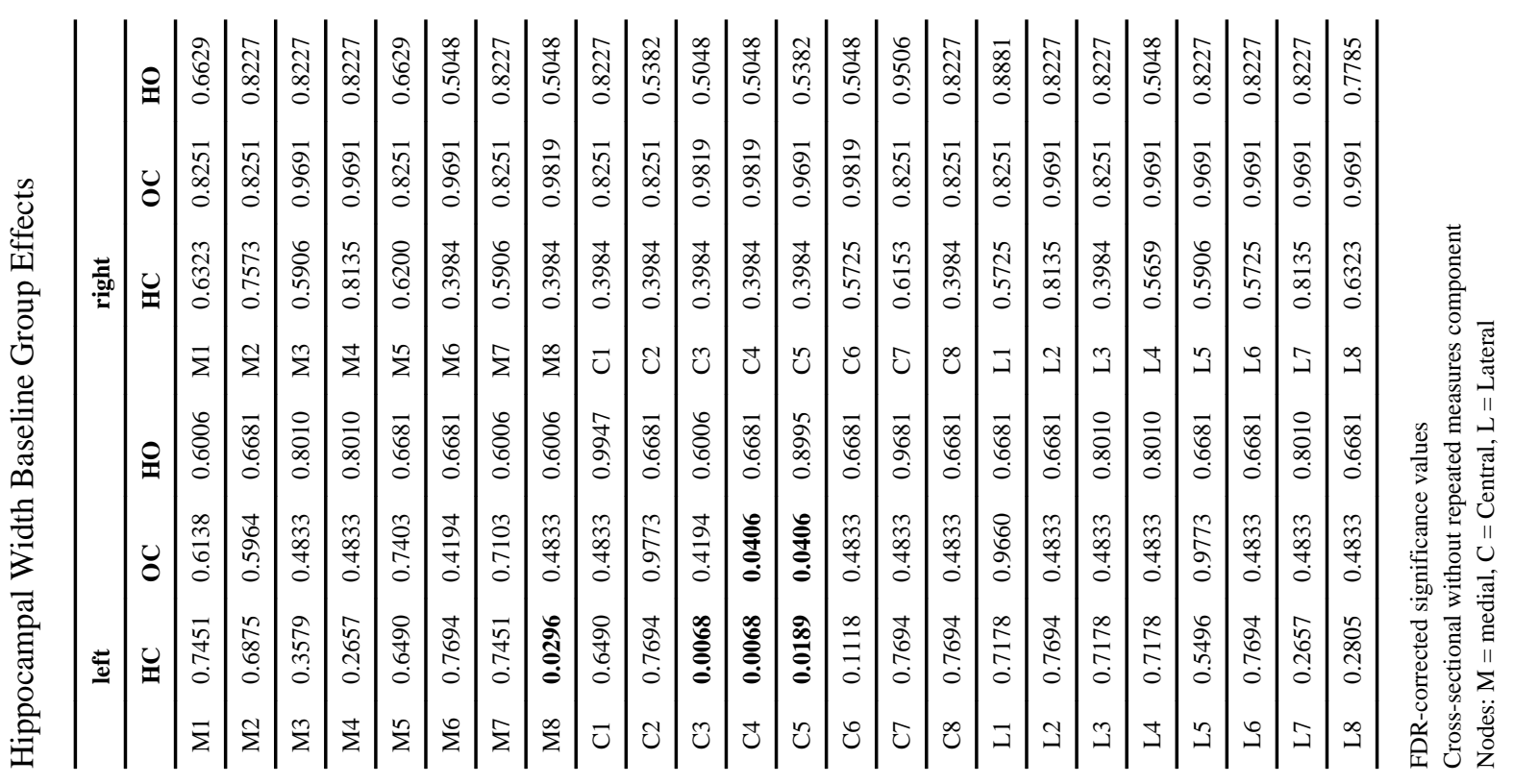




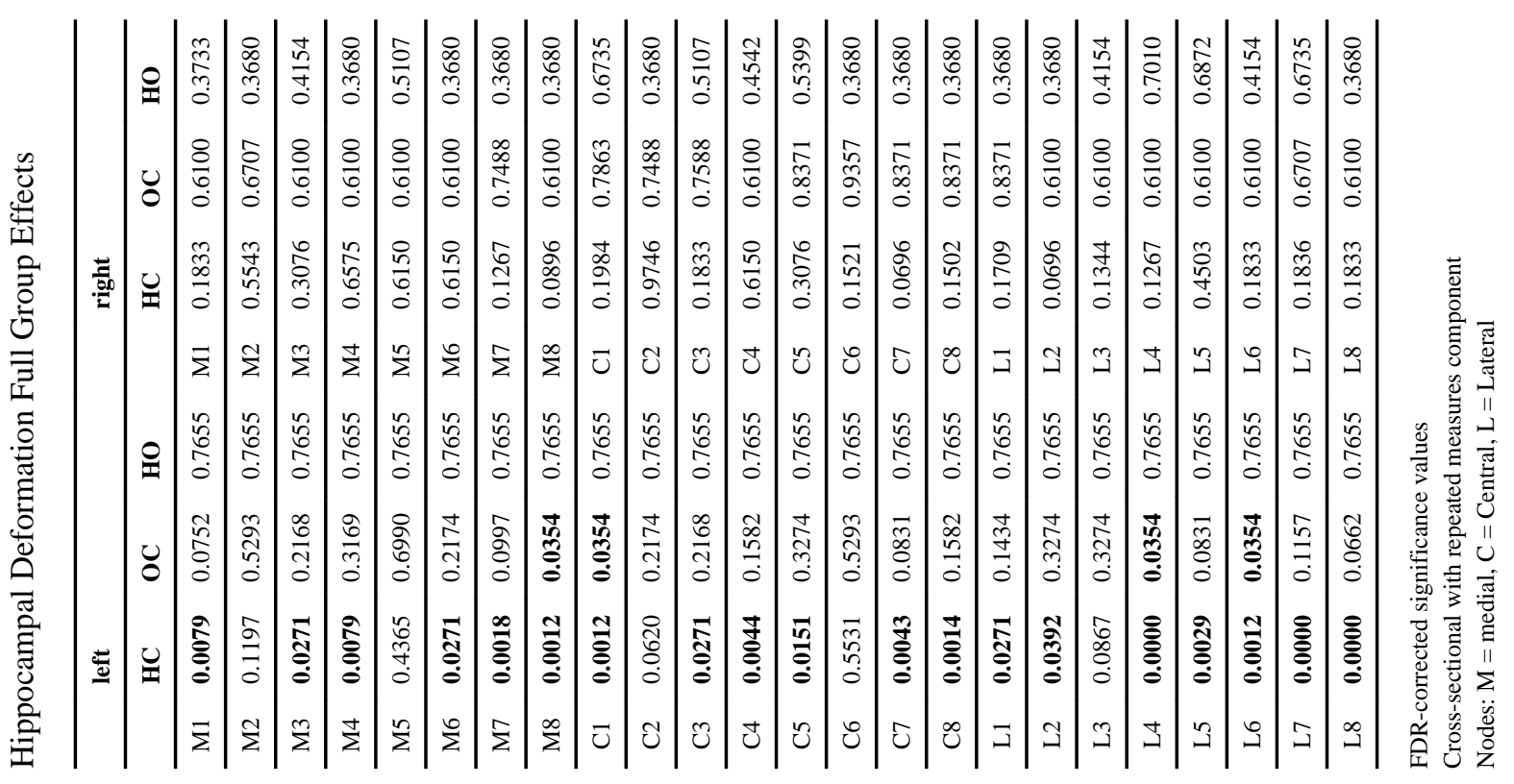




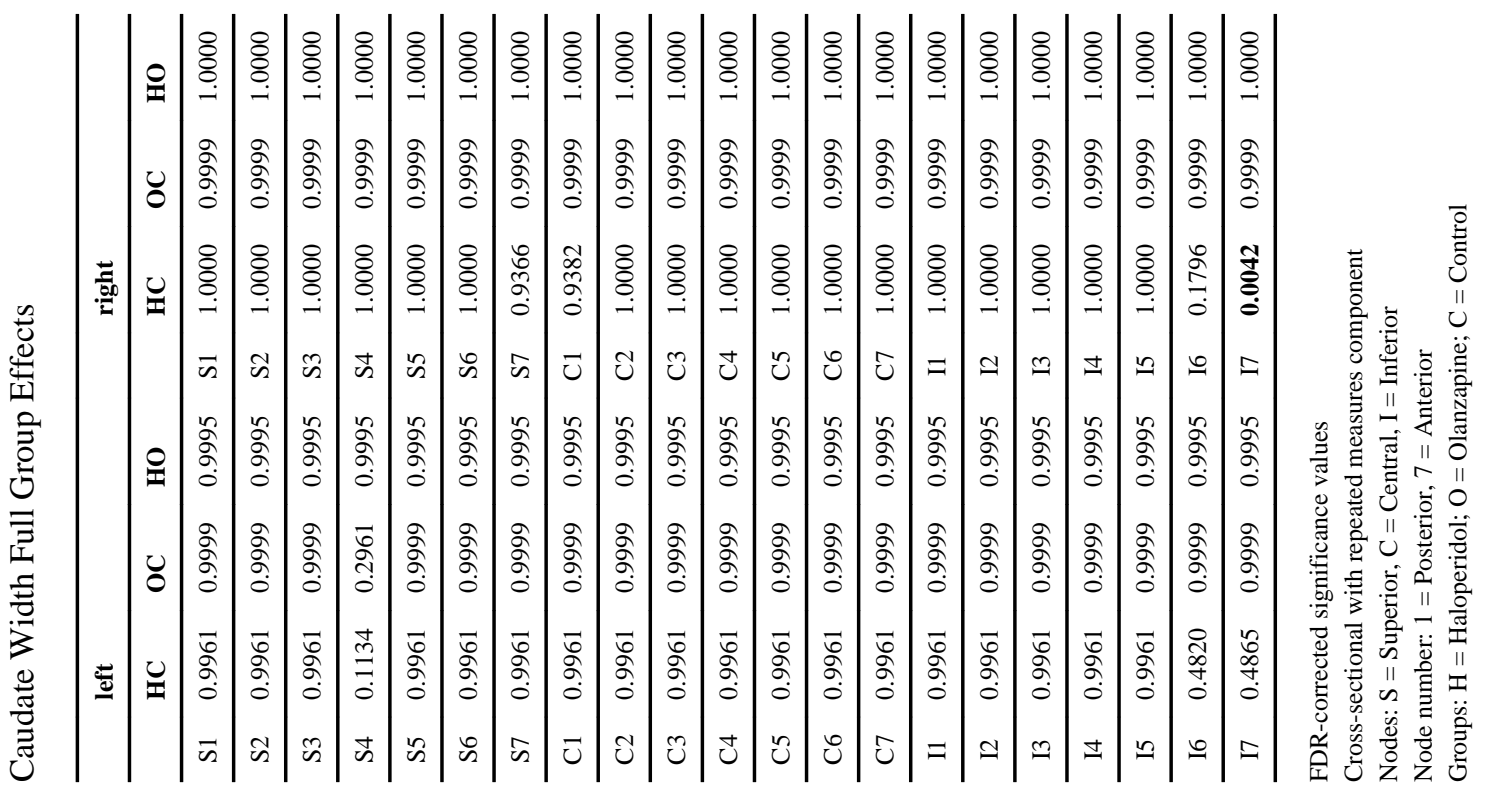




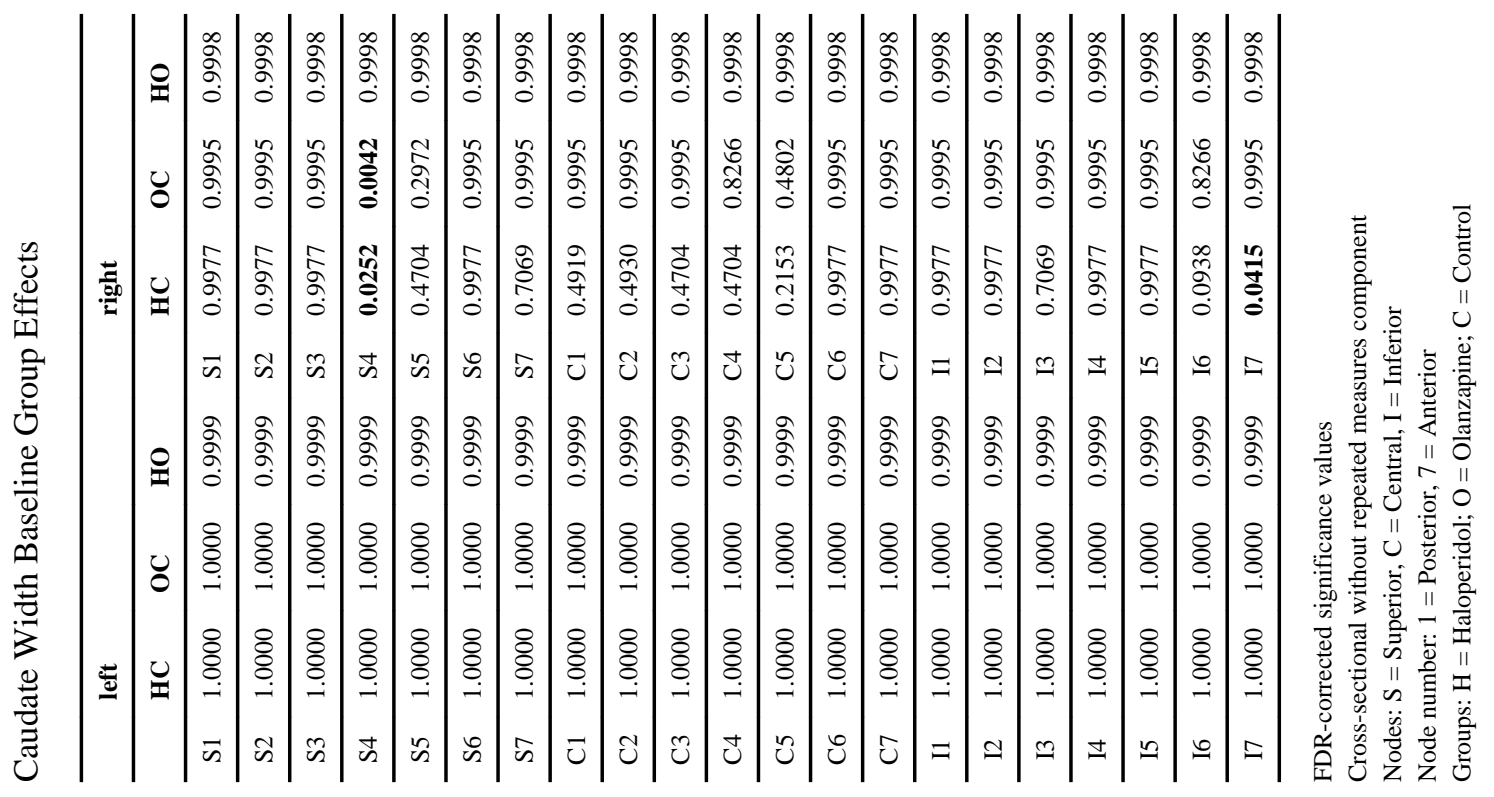




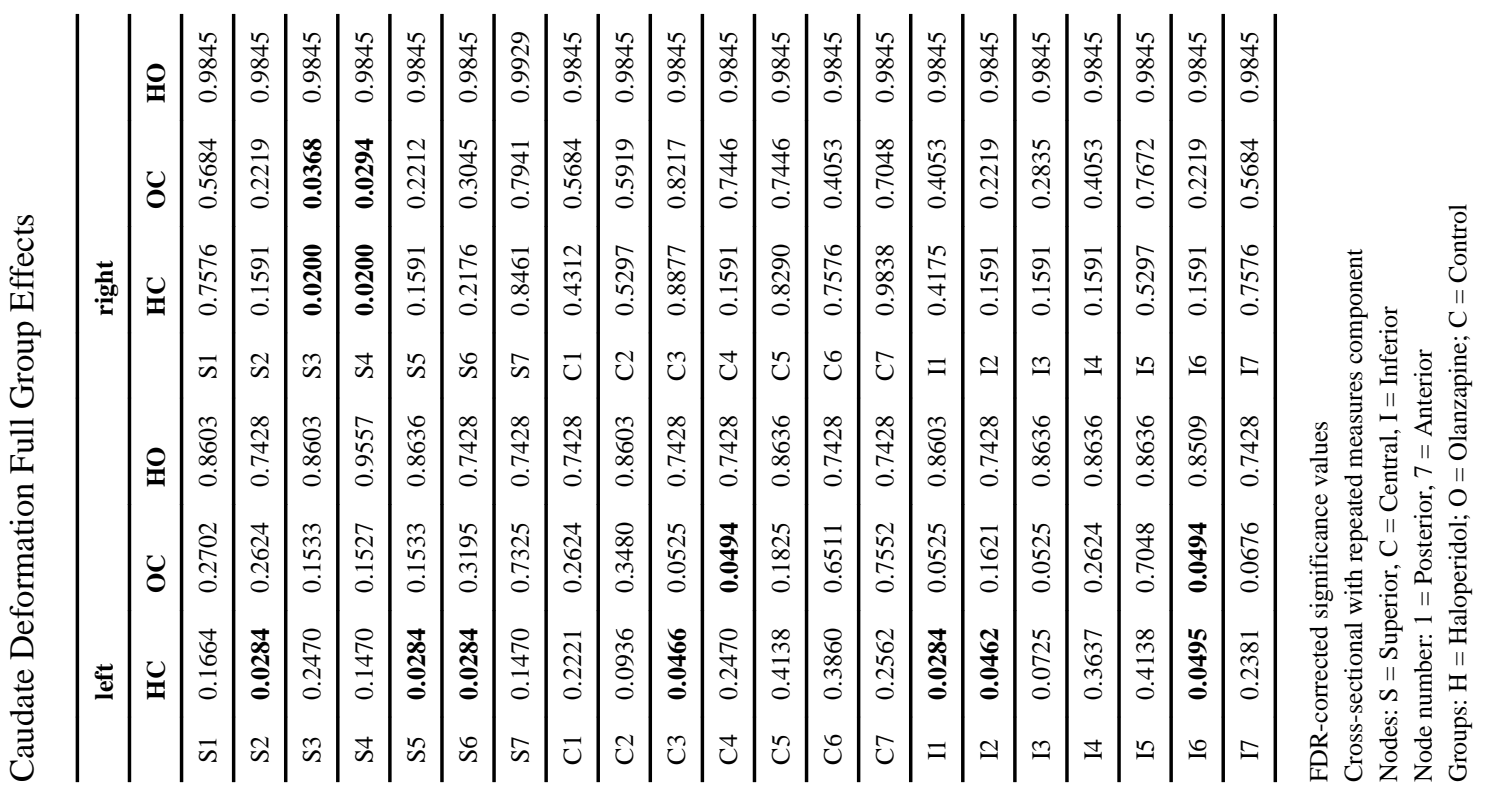


